\title{
Music-based interventions in neurological rehabilitation
}

\author{
Sihvonen, Aleksi J.
}

2017-08

Sihvonen , A J , Särkämö , T , Leo , V , Tervaniemi , M , Altenmueller , E \& Soinila , S 2017 ,

' Music-based interventions in neurological rehabilitation ', Lancet Neurology, vol. 16 , no. 8

, pp. 648-660 . https://doi.org/10.1016/S1474-4422(17)30168-0

http://hdl.handle.net/10138/311671

https://doi.org/10.1016/S1474-4422(17)30168-0

cc_by_nc_nd

acceptedVersion

Downloaded from Helda, University of Helsinki institutional repository.

This is an electronic reprint of the original article.

This reprint may differ from the original in pagination and typographic detail.

Please cite the original version. 


\section{Music-based interventions in neurological rehabilitation}

Aleksi J. Sihvonen, MD (corresponding author)

3 Faculty of Medicine, University of Turku, Finland

4 Cognitive Brain Research Unit, Department of Psychology and Logopedics, Faculty of Medicine, University

5 of Helsinki, Finland

6 Address: Cognitive Brain Research Unit, Department of Psychology and Logopedics, Faculty of Medicine,

7 Siltavuorenpenger 1 B, FI-00014 University of Helsinki, Finland

8 Email: ajsihv@utu.fi Phone: +358 405209386

9 Teppo Särkämö, PhD

10 Cognitive Brain Research Unit, Department of Psychology and Logopedics, Faculty of Medicine, University

11 of Helsinki, Finland

12 Vera Leo, MA

13 Cognitive Brain Research Unit, Department of Psychology and Logopedics, Faculty of Medicine, University

14 of Helsinki, Finland

15 Mari Tervaniemi, PhD

16 Cognitive Brain Research Unit, Department of Psychology and Logopedics, Faculty of Medicine, University

17 of Helsinki, Finland

18 CICERO Learning, University of Helsinki, Finland

19 Eckart Altenmüller, MD, $\mathrm{PhD}$

20 Institute of Music Physiology and Musicians' Medicine, University of Music and Drama Hannover, Germany

21 Seppo Soinila, MD, PhD

22 Division of Clinical Neurosciences, Turku University Hospital and Department of Neurology, University of

23 Turku, Finland

\section{Summary}

25 During the last ten years, an increasing number of controlled studies have addressed the 26 rehabilitative effects of music-based interventions in several neurological diseases. While

27 the amount of the studies and the level of evidence is highest in stroke and dementia,

28 increasing evidence is accumulating for the effects of music-based interventions in

29 Parkinson's disease, epilepsy, and multiple sclerosis. Studies have confirmed that

30 interventions, such as music listening, singing, or playing an instrument, are beneficial for

31 cognition, motor function, or emotional well-being in these patients. Although music-based

32 interventions may target divergent functions, such as motor performance, speech, or

33 cognition, the psychological effects and neurobiological mechanisms underlying the impact 
34 of music are likely to share common neural systems for reward, arousal, affect regulation, 35 learning, and activity-driven plasticity. Although further controlled studies are still needed 36 to establish the clinical efficacy of music in neurological recovery, music-based

37 interventions are emerging as promising rehabilitation strategies.

\section{Introduction}

39 The population is ageing rapidly and the number of persons suffering from severe agerelated brain diseases is rising ${ }^{1}$. Less than $20 \%$ of the heavy economic burden of chronic

41 brain diseases is due to acute treatment and care ${ }^{2,3}$. This has raised the need to pursue new cost-effective, light-input rehabilitation strategies, both independent of and complementary to traditional methods, such as physiotherapy, occupational therapy, or speech therapy.

Since neurogenesis in the adult brain has no clinically meaningful impact, brain recovery relies upon the spared neurons' ability to compensate for lost function by growing neurites and forming novel synapses to rebuild and remodel the injured networks ${ }^{4-8}$. This is thought to be achieved in traditional rehabilitation strategies by targeted training of the weakened function ${ }^{9-12}$. An alternative strategy would be to increase the overall level of brain activity through sensory and cognitive stimulation ${ }^{13}$.

51 Music listening improves neuronal connectivity in numerous specific brain regions of the healthy participants ${ }^{14-17}$, and musical activities, such as playing an instrument, promote neural plasticity, and induce changes in the grey matter and white matter ${ }^{18-20}$. Music has been shown to be efficacious in the recovery of postoperative patients by several outcome measures such as pain, anxiety, use of analgesics, and patient satisfaction, ${ }^{21}$ suggesting that music might enhance neurological rehabilitation as well.

57 Formal music-based intervention, music therapy, can comprise of active interventions (e.g.

58 music creating, instrument playing, singing, musical improvisation) and receptive 59 interventions (e.g. music listening) administrated by a credentialed music therapist.

60 Although a Cochrane review evaluating the effect of music interventions in acquired brain

61 injury has been recently published ${ }^{22}$, a comprehensive overview on music-related

62 interventions in the rehabilitation of the major neurological diseases, including

63 degenerative diseases and other neurological entities in which the rehabilitative effect of

64 music has been studied, is needed. Here, we appraise the randomized controlled trials 
65 (RCTs) investigating the effects of music-based interventions in the rehabilitation of stroke, 66 dementia, PD, epilepsy, and MS.

\section{Search strategy and selection criteria}

68 We searched PubMed up till April 11, 2017 using Medical Subject Headings (MeSH) for diseases "stroke", "brain injuries", "dementia”, "parkinsonian disorders", "epilepsy", and "multiple sclerosis", combined with MeSH for "music" or "music therapy" and keywords "melodic intonation therapy", "rhythmic auditory stimulation", "rhythmic auditory cueing", and "music supported therapy". Additional references were gathered from reference lists and relevant articles. We included only the RCTs applying a minimum of one-week intervention, published in English over the past 10 years, except for two older landmark studies.

\section{Music-based interventions for stroke}

Stroke is the one of the leading causes of long-term disability in the world ${ }^{23}$. Of the major

neurological entities, the strongest evidence for effectiveness of music-based interventions has been presented for stroke. We identified 16 RCTs utilizing music during recovery from stroke-related neurological and neuropsychiatric disturbances (Table 1) ${ }^{24-39}$. The parameters assessed included motor functions, such as gait and upper extremity function $24,25,27,30,33-39$, language functions ${ }^{26,28,29}$, cognitive functions, such as memory and attention $28,32, \operatorname{mood}^{28,32,34}$, or quality of life $(\mathrm{QoL})^{30,34}$. The measurements were carried out with various standard motor tests (e.g., Fugl-Meyer assessment, the Box and Block Test, Berg Balance Scale, and Nine-Hole Pegboard Test), clinical neuropsychological assessments (e.g., CogniSpeed, Wechsler Memory Scale), standard language function assessments (e.g., Boston Diagnostic Aphasia Examination), and questionnaires (e.g.,

88 Stroke Impact Scale, Profile of Mood States, and Stroke and Aphasia QoL Scale-39). In addition, computer-based movement analyses ${ }^{30,35,37,38}, \mathrm{MRI}$ analysis ${ }^{28,32,39}$, magnetoencephalography ${ }^{31}$, or electroencephalography ${ }^{33}$ were utilized to assess motor performance and neuroplasticity. Metronome-like rhythmic stimulus was used in five studies on stroke-related motor paresis ${ }^{30,34,36,37,39}$. Favorite music selected through interview was used in three studies ${ }^{28,31,32}$. The genres of favorite music were not reported.

94 Three studies used children's songs and folk songs ${ }^{24,33,35}$. Five studies involved a trained music therapist ${ }^{26-28,31,32}$. 


\section{Effects on motor symptoms}

97 Hemiparesis is the most common consequence of stroke, affecting over $70 \%$ of the patients ${ }^{40}$. In total, eight studies reported enhanced motor recovery when stroke patients were rehabilitated with music ${ }^{25,27,30,33-37}$. Four of these studies investigated the use of rhythmic auditory stimulation (RAS) in gait training and all found it to improve gait parameters more than gait training without any musical support ${ }^{30,36-38}$. In RAS, external auditory cues guide movement through anticipated temporal sequence, the frequency of which is adjustable and gradually entrains the movement. Across studies, significant improvements with small (Cohen's $d \geq 0.2$ ), medium ( $d \geq 0.5)$, or large ( $d \geq 0.8$ ) effect sizes (Table 1) were observed in gait velocity, stride length, length of foot contact to surface, cadence, and asymmetry after 3-6 weeks of RAS compared to conventional training without $\mathrm{RAS}^{36-38}$. Similar findings were reported when intensive gait training with RAS was investigated with respect to postural control and gait performance in chronic stroke patients $^{30}$. In 6 weeks, RAS group improved in balance, gait velocity, cadence, stride length, and double support period on the affected side ${ }^{30}$. When RAS was utilized in the form of combining rhythmic music with movement therapy, stroke patients showed improved ankle and arm movement after 8 weeks of intervention, with medium and large effect sizes, respectively ${ }^{34}$. One study compared bilateral arm training with RAS to dosematched therapeutic exercises, but found no significant differences between the groups ${ }^{39}$. Interestingly, RAS intervention conducted by a music therapist resulted in greater improvement compared to studies conducted by a non-music therapist ${ }^{22}$.

Music-supported therapy (MST), in which musical instruments (electric drum pads and keyboards) are used to train gross and fine movements of the hemiparetic upper extremity by playing simple melodies, was found to be effective in rehabilitating the arm paresis after stroke in five RCTs $24,25,27,33,35$. Three weeks of MST improved motor skills of the paretic arm significantly more than conventional physiotherapy, an effect shown by several validated clinical tests with small to medium effect sizes ${ }^{33,35}$. The effects were accompanied by improved cortical connectivity and increased activation of the motor cortex $^{33}$. These effects seem to be specifically caused by music rather than motor training per se, since patients practicing with mute instruments remained inferior to the music group $^{27}$.

One study utilized movement sonification therapy, a recent development in $\mathrm{MST}^{25}$. Gross movement was transformed into sound, providing continuous feedback, substituting for 
defective proprioception. Sonification therapy reduced joint pain and improved smoothness of movement more than movement therapy without sound with large effect sizes. Delayed auditory feedback in MST has been proposed to be as effective as the traditional immediate auditory feedback ${ }^{24}$. While both RAS and MST involve auditory-motor coupling, incorporating full music stimulus might result in additional enhancement due to the personal motivational value of music. Internal synchronization, based on musical memory, generates expectation of consecutive sounds of a familiar song and provides precise mental timing feedback for movement, thus supporting the patient's impaired proprioception.

\section{Effects on aphasia}

Aphasia affects around $30 \%$ of stroke patients ${ }^{40}$. In two RCTs, active music therapy improved the speech of chronic aphasics ${ }^{26,29}$. In one of them, Melodic Intonation Therapy $(\mathrm{MIT})^{41}$, a singing-based speech therapy designed for non-fluent aphasics, was applied on subacute aphasics ${ }^{29}$. MIT is a formalized treatment to transform the prosody of speech into low and high pitches - which the patient then learns to use to intone the stressed and nonstressed syllables, respectively - accompanied by rhythmic tapping with the left, nonparetic hand on each syllable. Training starts with two-syllable words and proceeds gradually to phrases. MIT improved the daily life communication and object naming significantly more than the control group receiving other types of language rehabilitation with medium and large effect sizes, respectively ${ }^{29}$. Music-related speech therapy, MIT in particular, is conceptually elegant and music therapy interventions may be more effective in aphasia than speech training without music 22.

\section{Effects on cognitive and emotional deficits}

Deficits in cognitive functions (e.g., memory, attention, executive function) and mood (e.g. depression) affect around $30-50 \%$ of stroke survivors ${ }^{42,43}$. In one RCT, one-hour daily listening to favorite music selected with the help of a music therapist and continued during the first two post-stroke months enhanced cognitive recovery ${ }^{32}$. In a 6-month follow-up, the music group still showed significant improvements with large effect sizes in performance of tasks measuring verbal memory and focused attention compared to a control intervention (audio book listening) or standard care ${ }^{32}$ (see Figure 1A). Compared to standard care, music listening was also associated with less depression and confusion with medium effects $^{32}$ (see Figure 1A). The cognitive gains induced by music listening were associated 
with enhanced auditory memory-related function in temporal brain areas ${ }^{31}$ and increased gray matter volume in spared prefrontal regions ${ }^{28}$ (Figure 1B-C). Music-induced reduction in negative mood was linked to increased grey matter volume in limbic areas ${ }^{28}$. In addition to music listening, RAS therapy improved patients' mood but with non-significant effect size $^{34}$. Although the long-lasting positive effects were shown by several outcome measures, these effects need to be replicated.

\section{Music-based interventions for dementia}

The most common etiologies of dementia are Alzheimer's disease, cerebrovascular diseases, and their combination. In these entities, neural degeneration progresses over several years leading sequentially to memory problems and other behavioral disturbances. Altogether 17 RCTs on persons with dementia (PWDs; Table 1) have assessed the effects of music intervention on neuropsychiatric and behavioral symptoms, such as anxiety, and agitation (14 studies) ${ }^{44-57}$, depression (six studies) ${ }^{47,49,55,58-60}$, cognitive status (five studies) $)^{47,49,51,58,59}$ as well as on QoL (four studies) $46,47,59,60$. Neuropsychiatric and behavioral symptoms were assessed with tests, rating scales, or questionnaires measuring overall symptom severity (e.g., Neuropsychiatric Inventory (NPI), CohenMansfield Agitation Inventory, Behavior Pathology in Alzheimer's Disease Rating Scale), depression (e.g., Cornell Scale for Depression in Dementia, Geriatric Depression Scale), cognitive status [e.g. Mini-Mental State Examination (MMSE), Severe Impairment Battery), and QoL or well-being (Cornell-Brown Scale for QoL in Dementia, Dementia Care Mapping). Most interventions used vocal or instrumental music presumably familiar to the PWDs, such as personal favorites, all-around popular music or common children's songs. All studies except for one involved a music therapist.

\section{Effects on cognitive deficits}

Music listening coupled with cognitive elements (reminiscence, attention training) or physical exercise improved overall cognitive performance (measured by MMSE) of patients with dementia compared to standard care in four studies published by three separate groups ${ }^{47,51,58,59}$. The effect sizes varied from small to medium. In addition, improved performance in these music interventions was reported for tests measuring attention and executive functions (small to medium effect size) ${ }^{51,59}$, orientation (medium effect size) ${ }^{59}$, and verbal or episodic memory (medium effect size) ${ }^{51,59}$. In one RCT, also caregiver-implemented singing was found to enhance working memory with medium effect 
size, especially in mild dementia and also to reduce caregiver burden as shown by a large effect size ${ }^{59}$. On the contrary, no significant changes in cognitive performance were observed for group-based music and cooking interventions in persons with moderatesevere dementia ${ }^{49}$. The cognitive benefits of music in the early stages of dementia may be related to enhanced cognitive reserve, the utilization of alternative networks and cognitive strategies to cope with advancing pathology ${ }^{61}$.

\section{Effects on neuropsychiatric symptoms, mood, and quality of life}

Six studies found music therapy to be effective in improving the neuropsychiatric symptoms of dementia with medium to large effect sizes ${ }^{44,46-48,54,56}$. Three studies assessed the carry-over effect $44,55,58$, which varied from less than four weeks to two months. In contrast, two studies failed to show any significant effect of music therapy or music listening on neuropsychiatric symptoms ${ }^{45,49}$. The music intervention program resulted also in improved PWD-caregiver interaction and well-being of the PWDs (large effect size $)^{46}$. Regarding specific neuropsychiatric symptoms, two studies showed music to reduce anxiety and agitation in PWDs ${ }^{52,55}$, but their effect sizes diverged. In contrast, four RCTs found music to be ineffective in reducing anxiety or agitation $50,51,53,57$.

QoL was assessed in three studies ${ }^{47,59,60}$. While Cooke et al. (2010) did not find any significant differences between the effects of music and control (reading) interventions ${ }^{60}$, Särkämö et al. (2014) reported that music listening compared to standard care increased QoL significantly and with large effect size ${ }^{59}$. Music listening was found especially beneficial in moderate dementia with etiology other than Alzheimer's disease ${ }^{47}$. Improvement of mood in PWDs has been reported in four studies, effect sizes varying between small and large $47,55,58,59$. Two other RCTs failed to show such an effect ${ }^{49,60}$. Overall, the effects of musical interventions in dementia may be driven by the comfort and emotional safety induced by familiar music, which can temporarily overcome the confusion and disorientation by anchoring attention on a positive familiar stimulus in an otherwise confusing environment. This anchoring effect may be enhanced by using headphones. Familiar music is also imbued with personal emotions, which can trigger autobiographical memories and help to restore a sense of identity for a while.

\section{Music-based interventions for Parkinson's disease}

Parkinson's disease (PD) is primarily a movement disorder due to degeneration of dopaminergic nigro-striatal tract. In addition, the early phase of PD includes autonomic 
nervous system and other non-motor deficits, and 30\% of the patients develop dementialevel cognitive decline in the late phase ${ }^{62}$. Effects of music on several symptoms and signs of PD have been studied in five RCTs (Table 1) ${ }^{63-67}$. Four studies examined the effects of music-assisted motor training using motor parameters as outcome measures ${ }^{63-66}$. Two studies $^{63,67}$ evaluated non-motor parameters, QoL, cognition, or social parameters. In all trials, medication remained unchanged during the interventions.

231 General motor performance was assessed by motor part of the Unified Parkinson's 232 Disease Rating Scale (UPDRS-III), and specific motor functions by e.g. Berg Balance 233 Scale and 6-minute walk test. Specific gait parameters were analyzed using video recordings and computer-assisted motion analysis programs. QoL was evaluated using validated questionnaires. Music used in the intervention varied from rhythmic auditory cueing to self-selected favorite music. The genres of the patient's favorite music were not reported. Only one study involved a music therapist ${ }^{63}$.

238 Based on effect sizes calculated from the reviewed data, the most coherent and clinically 239 significant beneficial effect on motor symptoms was produced by dancing. Compared to 240 the standard care, both tango and waltz or foxtrot intervention groups improved in balance, 2416 -minute walk test, and backward stride length with large effect sizes ${ }^{65}$. In a smaller study, 242 tango improved balance with large effect ${ }^{66}$. Dancing also improved overall mobility with 243 large effect size ${ }^{67}$. Bearing a close analogy to dancing, music therapy with rhythmic 244 movements ${ }^{63}$ improved overall mobility in patients with PD. Gait training synchronized to 245 music resulted in improved velocity, stride time, and cadence with large effect sizes 246 compared to the control group ${ }^{64}$. Both studies reported reduction in PD specific motor 247 symptoms (medium effect size) $63,64$.

248 Two studies found music-based intervention to improve QoL with large effect size $63,67$. 249 Dancing tango appeared to be significantly more effective than waltz, Tai Chi or regular 250 treatment ${ }^{67}$. In addition, patients reported better social support after the intervention. 251 Improvements in cognition have been reported in one study ${ }^{63}$.

252 Although the sample sizes were relatively small, the reviewed evidence suggest that 253 dancing and music-based interventions that synchronize movement to music can be 254 beneficial in maintenance of motor performance in this slowly progressing disease. 255 Rhythmical use of musical stimulus compensates for the failing control by the extrapyramidal system and enhances audio perception and movement 
synchronization $30,36,37$. The perceived rhythm in music activates the neural circuits involved in motor actions and act as an external cue for movement thus replacing the impaired internal timing function in $\mathrm{PD}^{68}$. The use of music as stimulus may be more effective than auditory stimulation without music (e.g. metronome beat) in gait rehabilitation, as shown in stroke $^{22}$. This might also explain the positive effects of dancing in PD. Furthermore, the improvement in motor control and possible decrease in disease specific symptoms could in turn improve the QoL. In all of studies reviewed, the follow-up period was too short to allow conclusions on the long-term effects of music interventions. The effects of music on the autonomic disturbances in PD have not been addressed in controlled studies.

\section{Music-based interventions for multiple sclerosis}

Multiple sclerosis (MS) is one of the most common severe neurological disease in the young adult population. Despite relatively low prevalence, it bears need for expensive medication and long-lasting rehabilitation ${ }^{3}$. MS treatments aim to ameliorate function after flare-up of an MS-episode or to prevent new episodes. Only two RCTs ${ }^{69,70}$ (Table 1) have studied the effect of musical interventions in alleviating the manifestations of MS. Between the studies, outcomes were different, and only one study involved a music therapist.

The RCT without music therapist included 19 patients and studied the effect of keyboard playing (audible vs. mute) in hand functionality ${ }^{69}$. Audible keyboard playing improved the functional use of the hand significantly with medium effect size, indicated by a questionnaire. Using a computerized gait analysis, a feasibility study on ten MS patients with gait problems found RAS to be effective in decreasing double-support time with large effect size ${ }^{70}$. While decreased double-support time may reflect improved dynamic balance ${ }^{71}$, none of the several other gait parameters differed from controls receiving standard care. The results of music-based interventions in MS are scanty and allow no definite conclusions on the rehabilitative effect of music. Although designing studies may be challenging due to diversity of MS deficits, motor functions, spasticity, fatigue, cognitive deficits, and mood might be feasible outcome measures in the future studies.

\section{Music-based interventions for epilepsy}

285 Epileptic seizures arise from abnormal synchronization of electrical activity in the brain, and the most of them cease spontaneously by largely unknown mechanisms. Exposure to 
288 has been suggested to reduce epileptiform activity ${ }^{72}$. In this vein, one RCT ( $N=73$; Table 1) 289 has examined the effectiveness of music in epilepsy ${ }^{73}$. Patients were exposed to Mozart's 290 music periodically every night for a year and a significant $17 \%$ reduction in seizure 291 frequency was detected during the study period. In addition, a carry-over effect of $16 \%$ 292 reduced seizure frequency persisted for one year. While no other RCTs on adult 293 population have been published, a recent meta-analysis of 12 studies including both 294 pediatric and adult patients with epilepsy of any kind indicated that 130 out of 153 patients 295 respond favorably to music, the average reduction in interictal epileptic activity being $31 \%$ 296 and $24 \%$ during and after the listening period, respectively ${ }^{74}$. Further studies are definitely needed, since all but two studies lacked a separate control group.

\section{Mechanisms underlying the rehabilitative effect of music}

299 Specific pathologies of the diseases evaluated here may affect, sometimes critically, the way the patient's brain processes music, and diverse manifestations of the diseases influence the selection of feasible music intervention. Considering the widely varying nature of the diseases in which music has led to improved recovery, enhanced rehabilitation, or alleviation of symptoms, several distinct explanatory mechanisms can be postulated.

\section{Neural activation and neuroplasticity}

Functional neuroimaging studies have shown that music induces widespread activation of the brain ${ }^{14-17}$ (Figure 2), and correspondingly increases blood flow through the medial cerebral artery due to autoregulation ${ }^{75}$ (Figure 3 ). This should provide favorable circumstances for recovery processes in general regardless of their nature, as for example after stroke, neuroplastic changes associated with functional recovery are activitydependent $^{76}$. Musical activities bear similarity to the concept of enriched environment which facilitates recovery at behavioral and neurobiological levels in animal models of many neurological illnesses ${ }^{13}$.

314 Given that active music-based rehabilitation involves multiple components analogous to musical training and music learning (i.e. iterated practice of movements coupled with auditory feedback and extensive cognitive processing), it is plausible that music-based neurological rehabilitation induces similar structural and functional neuroplastic changes as musical training ${ }^{18,19}$. Indeed, individual studies have reported memory-related plastic effects after mere music listening in recovering stroke patients ${ }^{28,31}$ as well as neural 
reorganization after $\mathrm{MST}^{33}$. Supporting literature has provided further evidence of neuroplasticity after $\mathrm{MST}^{77-79}$ and $\mathrm{MIT}^{80}$ in stroke patients.

In general, the specific cellular mechanisms of neuroplasticity remain unknown. While significant neurogenesis in elderly individuals seems unlikely, other putative mechanisms include neuronal hypertrophy, increased volume of neuropil, and changes in the vascular or glial compartments. An intriguing question would be to investigate, whether previous music exposure during a specific period of lifetime affects the plasticity of recovering brain. The possibility of negative plastic changes due to overly intense and/or premature intervention should be considered.

\section{Activation of reward, arousal, and emotion networks}

Music activates the dopaminergic mesolimbic system which regulates memory, attention, executive functions, mood, and motivation ${ }^{81}$ (Figure 3). A key part of this reward system is the nucleus accumbens, which regulates mood and experienced pleasure. Its activation by intense emotional response ("chills") to music leads in healthy subjects to increased dopamine secretion directly proportional to the intensity of the experience ${ }^{81}$. This may partly explain the cognitive-emotional gains induced by music also in neurological patients. It is feasible to postulate that music-induced improvement of mood, arousal, and relief of confusion may enhance recovery of cognitive functions in neurological patients. Musicinduced activation of the parasympathetic and inhibition of the sympathetic nervous system in PWDs, and corresponding changes in catecholamine and cytokine secretion has been considered as a soothing effect of music ${ }^{82}$. This is also a possible mechanism behind the effect of music ameliorating neuropsychiatric symptoms in dementia.

Music also produces measurable cardiovascular and endocrine responses indicated by lowered serum cortisol levels and inhibition of cardiovascular stress reactions ${ }^{82,83}$ (Figure 3). In animal models, prolonged stress can have maladaptive effects on neuroplasticity, such as dendritic atrophy, synapse loss, and decreased hippocampal neurogenesis ${ }^{84} . \ln$ patients, elevated cortisol level in acute stroke correlates with increased infarct volume, and increases the risk of depression, poor prognosis, and fatal outcome ${ }^{85}$. We speculate that listening to music lowers stress hormone secretion in acute stroke, as it does in postoperative patients ${ }^{86,87}$. 
350 Overall, neurological diseases and mood disorders have a high comorbidity, ranging from $35120 \%$ to $50 \% 88,89$. Common clinical experience is that depression diminishes adherence to 352 rehabilitation, and published studies indicate that depression impairs functional outcome,

353 QoL, and increases mortality ${ }^{90}$. According to the data reviewed here, music improved 354 mood or diminished anxiety in PWDs ${ }^{52,59}$ and stroke patients ${ }^{32,34}$. We conclude that music 355 interventions are viable in improving the mood of neurologic patients. Yet, the causal 356 relationship between music-induced mood improvement and neurological outcome still 357 remains to be proved.

\section{Activation of alternative or spared neural networks}

359 Some music interventions allow access to an impaired function by engaging specific regions associated with musical rhythm, movement, singing, or memory ${ }^{68}$. Rhythmic entrainment, our inherent tendency to time movements to the regular beat of music, which forms the basis of RAS and playing-based music interventions, is based on the strong connectivity between the auditory system and motor system ${ }^{14}$. In diseases in which the internal sequencing and monitoring of actions is not working due to the dysfunction of the motor system, rhythmic entrainment can act as an external timer, cueing the execution of movements ${ }^{68}$. For instance, a stroke patient with impaired muscle coordination or a Parkinson patient with stiffness and bradykinesia may find it easier to execute motor tasks with rhythmic support provided by music listening or dancing $30,36-38,63-66$.

369 Singing, which is the key component of MIT, engages frontotemporal language and vocalmotor regions more extensively and bilaterally compared to speaking ${ }^{91,92}$. This enables

371 training of speech in aphasia via both spared left hemisphere regions and homologous 372 right hemisphere regions. The preserved ability to sing in aphasia has been reported as 373 early as 1745 , when a stroke patient with severe aphasia was reported to be only able to 374 verbalize "yes", but was able to correctly sing familiar hymns producing both the melody and the text of the songs ${ }^{93}$.

376 Familiar music specifically activates the anterior cingulate and medial prefrontal cortex in 377 the healthy brain, suggesting that they are important in musical memory ${ }^{94}$. In persons with 378 Alzheimer's disease, the medial prefrontal cortex degenerates more slowly than other 379 cortical regions and the regions that encode musical memory also show only minimal atrophy or decrease in glucose metabolism despite visible amyloid-beta accumulation ${ }^{94}$. 
381 These observations provide a potential explanation why Alzheimer patients are able to 382 recognize and respond emotionally to familiar songs even at late stages of the disease ${ }^{94}$.

\section{Conclusions and future directions}

Acute care and treatment accounts for a substantial proportion of costs associated with neurological diseases, and therefore, study of novel rehabilitation strategies to replace or complement traditional methods is warranted. With this aim, the effects of music-based rehabilitation in major neurological disorders have been studied in 41 RCTs. Music interventions seem to be beneficial particularly in motor rehabilitation in PD and stroke. Additionally, music interventions can have favorable effects on cognition, mood, and QoL in stroke and dementia.

Although the majority of the reviewed studies have reported positive effects, the possibility of publication bias should be considered. In addition, only few of the primary outcomes have been studied repeatedly. Limitations involved in most studies arise from small sample sizes and methodological heterogeneity in study design and in the interventions and outcome measures used across studies. In most studies, the duration of the musicinduced rehabilitation effect was not systematically evaluated and is still largely unknown. Thus far, music-based interventions have been observed to have long-term effects in stroke (3 months) ${ }^{32}$, dementia (max. 2 months $)^{44,55,58}$, and epilepsy (12 months) ${ }^{73}$.

In some studies, the difference between active and receptive intervention as well as the role of the music therapist (if participating) remained unclear. The therapeutic relationship inherent in formal music therapy is likely to have an additional impact on the outcome. While this aspect is difficult to delineate from the music intervention used, the outcome of an intervention given by a music therapist may in some cases be superior to that given by another health-care professional, as has been observed for RAS in gait rehabilitation ${ }^{22}$. However, the studies reviewed here showed that both music therapy and other musicbased interventions have beneficial effects. Most of the studies lacked adequate description of the music type used. As music types can greatly vary (e.g. stimulating vs. soothing), the expected effects on physiological parameters, arousal, and affect regulation differ. Furthermore, most of the reviewed studies did not use patient-selected or favorite music. Concerning the strong emotional components of musical experience, using patientselected music would be beneficial as it is meaningful and rewarding to the patient. 
412 More high-quality intervention studies, particularly large-scale trials, such as cluster413 randomized multicenter RCTs, where the established music interventions are embedded 414 into the clinical rehabilitation practice, would be needed to establish their efficacy and the 415 real-life applicability. For better comparability of the studies, it would also be important to 416 use common outcome measures, clearly document the type of the intervention (active vs. 417 receptive), and music used (patient-selected vs. experimenter-selected) as well as define 418 the optimal timing and length of the music interventions and determine the long-term 419 duration of their rehabilitation effects. In addition, multimodal studies combining behavioral 420 outcome measures with neuroimaging and neuroendocrinological markers are needed to 421 determine specific neurophysiological mechanisms and effects of various music-based 422 interventions in neurological patients.

423 Analysis of the amount of core therapeutic activities received, such as physiotherapy and 424 occupational therapy, suggests that stroke patients receive only approximately $60 \%$ out of 425 the recommended rehabilitation ${ }^{95}$. Scarceness of rehabilitation resources is likely to exist 426 in most neurological wards. Thus, there is room for music interventions that are widely 427 available and could easily be realized with minimal investments. These include self- or 428 caregiver-implemented musical activities, such as music listening, and group-based 429 musical interventions, such as group singing or dancing.

430 In future, mobile music applications (e.g., music streaming, games) as well as novel 431 music-based rehabilitation technology utilizing virtual reality or adaptive music stimulation 432 systems tailored for motor rehabilitation, will play an increasing role in bringing music to 433 neurological patients, in both hospital, community, and home environments.

\section{Contributors}

435 A.J.S, V.L., and S.S. searched and reviewed the literature, A.J.S. created figures and 436 tables, A.J.S. and S.S. wrote the primary manuscript, which was circulated among the 437 other authors T.S., E.A., M.T. and V.L. All made significant additions based on their 438 special areas of interest, which were incorporated into the final manuscript.

\section{Declaration of interests}

440 The authors have no conflict of interest to declare. 


\section{Acknowledgments}

442 T.S. received support from the Academy of Finland program (1277693), S.S. from the 443 Research Grants of Turku University Hospital (EVO 8140/2012, 2014), A.J.S. from the 444 Finnish Brain Research and Rehabilitation Foundation, Signe and Ane Gyllenberg 445 Foundation, and Maire Taponen Foundation, and V.L. from the National Doctoral 446 Programme of Psychology, Finland.

\section{Figures and tables}

448 Figure 1 Cognitive, emotional and neural effects of daily music listening (Music group), 449 audio book listening (Audio book group), and standard care (Control group) 1 week 450 (baseline, BL), 3 months ( $3 \mathrm{~m})$ and 6 months $(6 \mathrm{~m})$ after stroke. (A) Neuropsychological 451 results (mean \pm SEM) showing improved recovery of verbal memory and focused attention 452 (baseline score subtracted from the values) and less depression and confusion in the 453 Music group compared to the Audio book and Control groups. ${ }^{* *} P<0.01$, ${ }^{*} P<0.05$ by 454 mixed-model ANOVA. \#\#P $<0.05$, \#P $<0.1$ by one-way ANOVA. Adapted from Särkämö 455 et al. 2008. (B) Magnetoencephalography (MEG) group results (mean \pm SEM) showing 456 increased right hemisphere mismatch negativity (MMN) responses to pitch changes in the 457 Music and Audio book groups compared to the Control group. Adapted from Särkämö et 458 al. 2010. (C) Voxel-based morphometry (VBM) results of MRI data from left hemisphere459 damaged patients (lesion areas in blue-green) showing larger grey matter volume (GMV) 460 increases (mean \pm SEM) in prefrontal and limbic areas in the Music group compared to the 461 Audio book and Control groups. Results are shown at $p<0.01$ (uncorrected) with $\geq 50$ 462 voxels of spatial extent. $L=$ left hemisphere. Adapted from Särkämö et al. 2014. 


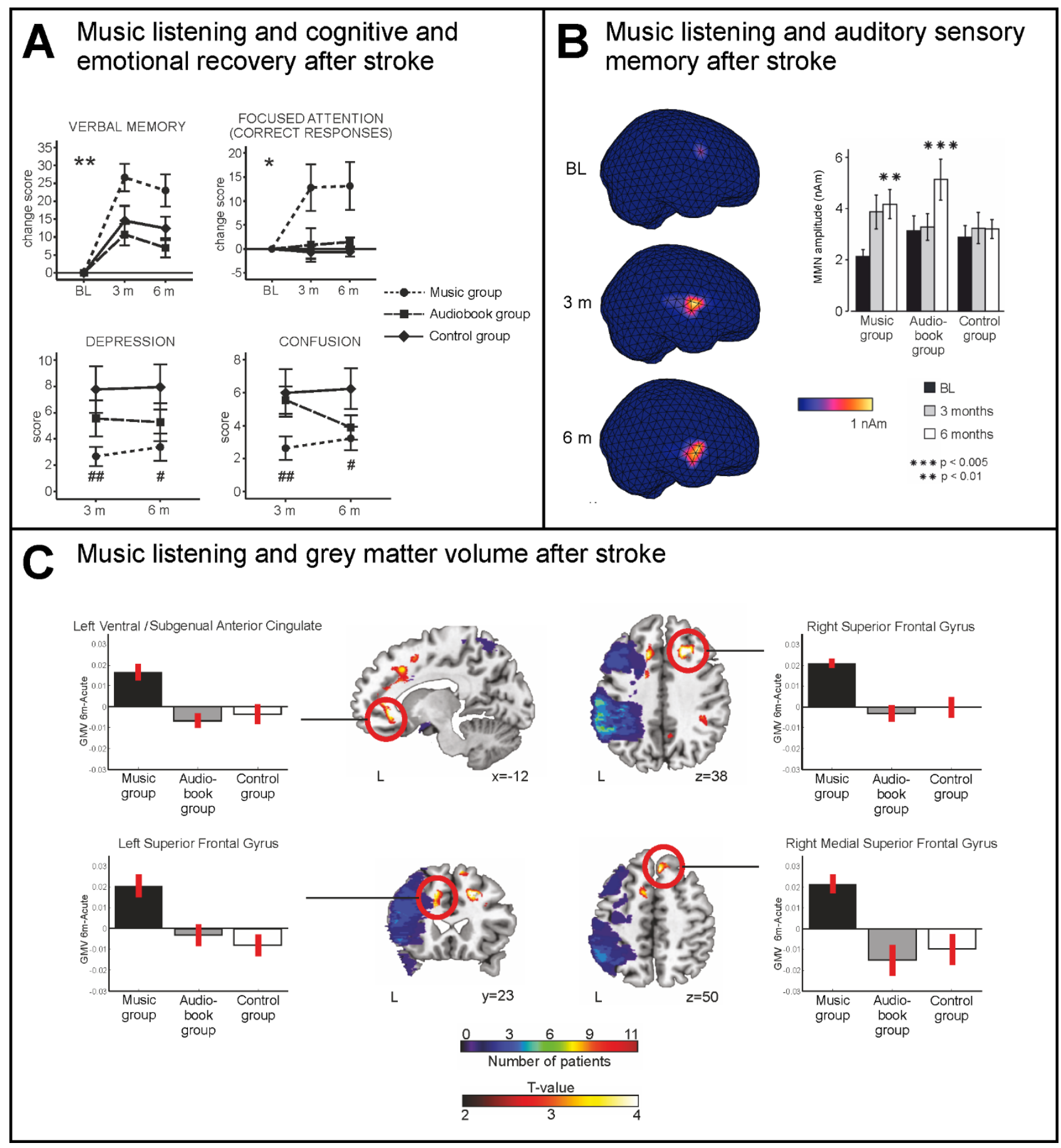

465 Figure 2 Schematic illustration of key brain areas associated with music processing466 based neuroimaging studies of healthy subjects. Note that although the image displays the 467 lateral and medial parts of the right hemisphere, many musical subfunctions are actually 468 largely bilateral (with the exception of pitch and melody processing, which are lateralized, 469 the activity in the right hemisphere being dominant). Adapted from Särkämö et al. 2013. 


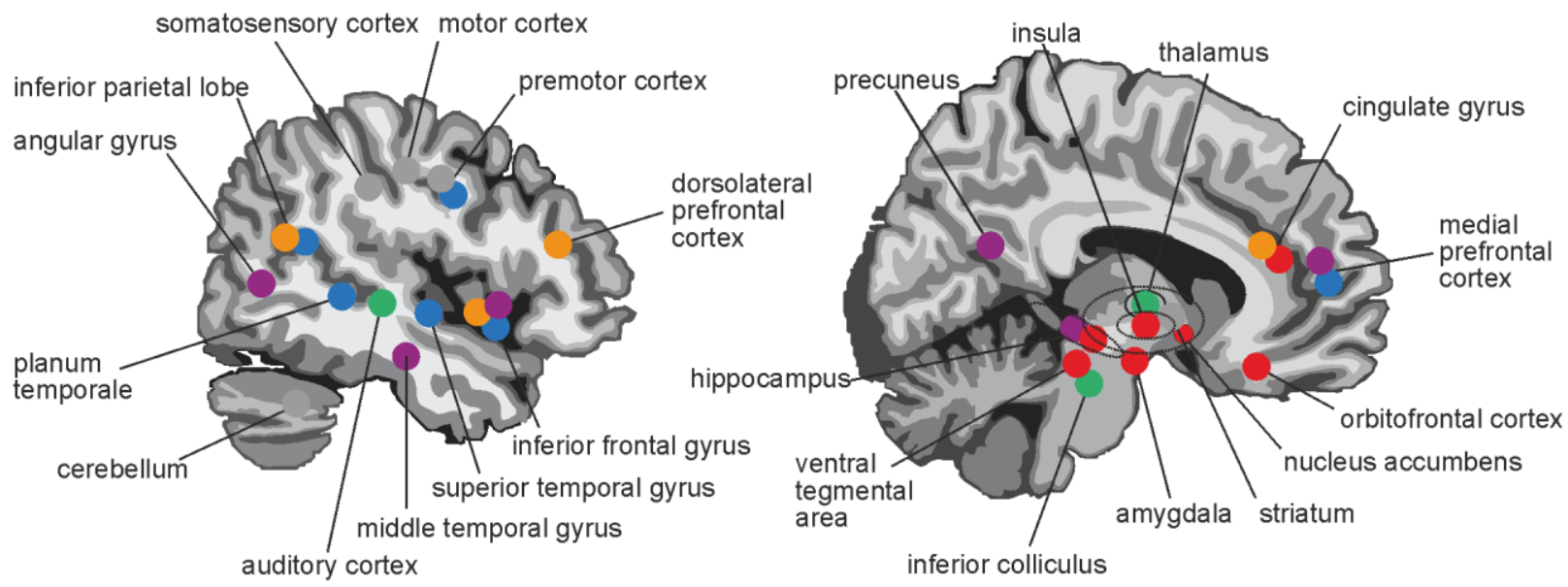

Basic auditory pathway: perceiving the basic acoustic features of music

Music-syntactic network: perceiving higher-order musical features

Attention and working memory network: focusing and keeping track of music in time

Episodic memory network: recognizing music and recalling associated memories

Motor network: playing, singing and moving to the beat of music

Reward and emotion network: music-evoked emotions and experiencing pleasure and reward

472 Figure 3 Schematic illustration of possible neurobiological mechanisms for underlying 473 the rehabilitative effect of music. Orange circles and yellow arrows represent the 474 mesolimbic system, and the green circles represent the hypothalamic-pituitary-adrenal 475 axis (HPA-axis). ACTH = Adrenocorticotropic hormone, CORT = Cortisol, $\mathrm{CRH}=$ 476 Corticotropin-releasing hormone. 


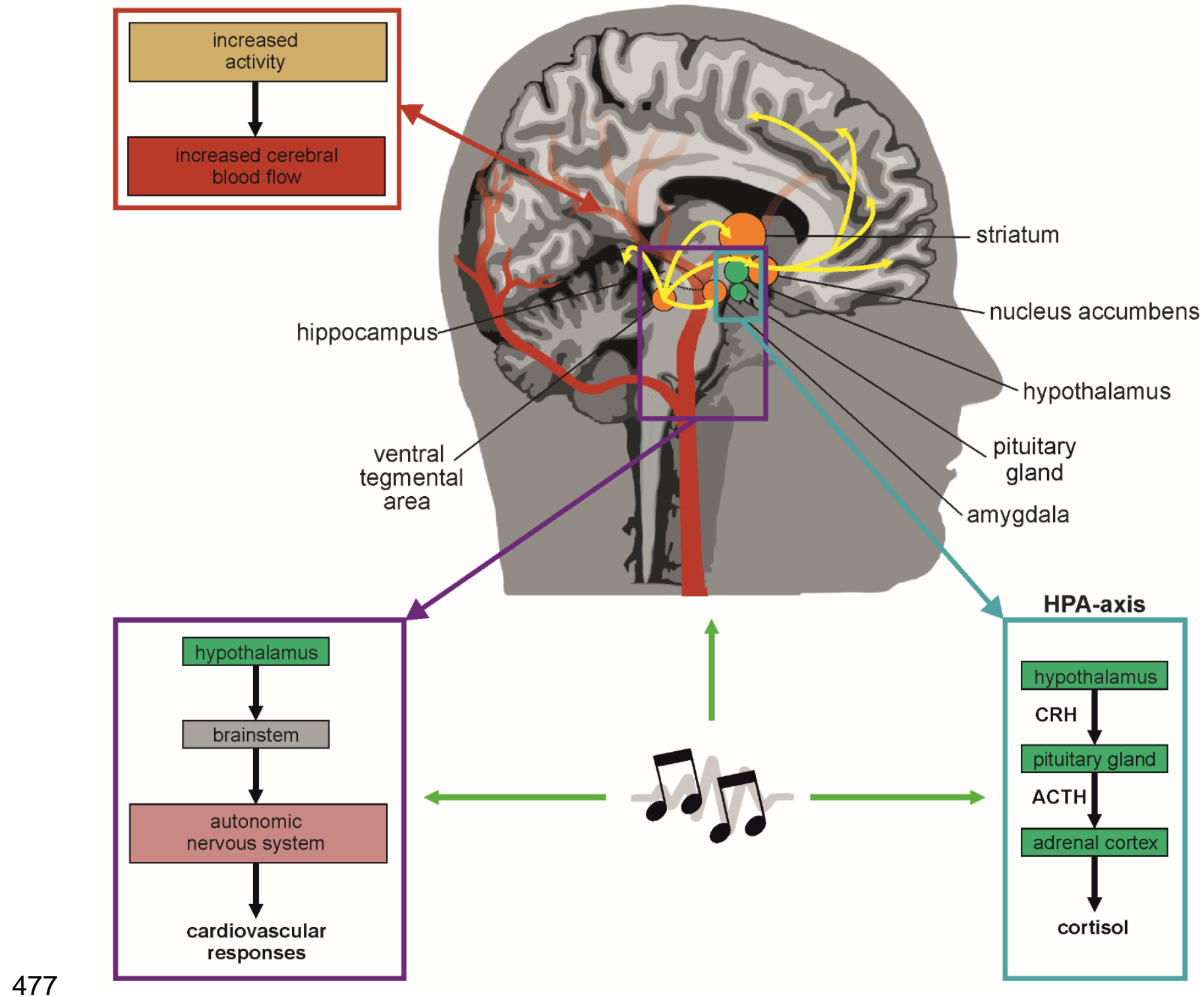




\section{STROKE}

\begin{tabular}{|c|c|c|c|c|c|c|}
\hline $\begin{array}{l}\text { van Vugt et al. } \\
(2016)^{24}\end{array}$ & 34 & No & Single & $\begin{array}{l}\text { MST vs. MST with delayed } \\
\text { sound / Hand movement. }\end{array}$ & $\begin{array}{l}5 \text { hours in } \\
4 \text { weeks }\end{array}$ & There were no significant differences between the groups. \\
\hline $\begin{array}{l}\text { Scholz et al. } \\
(2016)^{25}\end{array}$ & 25 & No & No & $\begin{array}{l}\text { Sonificated movement vs. } \\
\text { movement without sound / } \\
\text { Gross motor function. }\end{array}$ & 10 days & $\begin{array}{c}\text { Sonification therapy reduced joint pain }(p<0.05, d=1.96) \text { and improved } \\
\text { movement smoothness }(p=0.04, d=1 \cdot 16) .\end{array}$ \\
\hline $\begin{array}{l}\text { Raglio et al. } \\
(2016)^{26}\end{array}$ & 20 & Yes & No & $\begin{array}{l}\text { Singing, playing instruments, } \\
\text { improvisation vs. speech } \\
\text { therapy / Speech } \\
\text { parameters in chronic } \\
\text { aphasics. }\end{array}$ & $\begin{array}{l}22 \cdot 5-37 \cdot 5 \text { hours } \\
\text { in } 15 \text { weeks }\end{array}$ & Music therapy improved spontaneous speech $(p=0.020, d=0.35)$. \\
\hline $\begin{array}{l}\text { Tong et al. } \\
(2015)^{27}\end{array}$ & 33 & Yes & No & $\begin{array}{l}\text { MST vs. playing mute } \\
\text { instruments / Upper-limb } \\
\text { motor function. }\end{array}$ & $\begin{array}{l}20 \text { sessions } \\
\text { in } 4 \text { weeks }\end{array}$ & MST improved motor functions $(p=0.039)$. \\
\hline $\begin{array}{l}\text { Särkämö et al. } \\
(2014)^{28}\end{array}$ & 49 & Yes & Single & $\begin{array}{l}\text { Favourite music vs. standard } \\
\text { care / Grey matter changes } \\
\text { associated with cognitive } \\
\text { improvement. }\end{array}$ & $\begin{array}{l}60 \text { hours in } \\
8 \text { weeks }\end{array}$ & $\begin{array}{l}\text { Music listening increased gray matter volume in frontal areas, limbic areas, and } \\
\text { right ventral striatum. Reorganization in the frontal areas correlated with } \\
\text { enhanced recovery of verbal memory, focused attention, and language skills, } \\
\text { whereas the limbic area reorganization correlated with reduced negative mood. }\end{array}$ \\
\hline $\begin{array}{l}\text { n der Meulen et al. } \\
\qquad(2014)^{29}\end{array}$ & 27 & No & Single & $\begin{array}{l}\text { MIT vs. other language } \\
\text { intervention / Speech } \\
\text { parameters in nonfluent } \\
\text { aphasics. }\end{array}$ & $\begin{array}{l}30 \text { hours in } \\
6 \text { weeks }\end{array}$ & $\begin{array}{l}\text { MIT improved the daily life communication }(d=0.76) \text { and object naming }(d= \\
1 \cdot 73) .\end{array}$ \\
\hline $\begin{array}{l}\text { Cha et al. } \\
(2014)^{30}\end{array}$ & 20 & & No & $\begin{array}{l}\text { RAS vs. intensive gait } \\
\text { training / Postural control } \\
\text { and gait performance. }\end{array}$ & $\begin{array}{l}15 \text { hours in } \\
6 \text { weeks }\end{array}$ & $\begin{array}{l}\text { RAS improved balance, gait velocity, cadence, stride length and double support } \\
\text { period on the affected side, and in stroke-specific quality of life scale. }\end{array}$ \\
\hline $\begin{array}{l}\text { Whitall et al. } \\
(2011)^{39}\end{array}$ & 92 & No & Single & $\begin{array}{l}\text { BATRAC vs. normal } \\
\text { exercise / Functional } \\
\text { reorganization and outcome. }\end{array}$ & $\begin{array}{l}18 \text { hours in } \\
6 \text { weeks }\end{array}$ & There were no significant differences between the groups. \\
\hline $\begin{array}{l}\text { Särkämö et al. } \\
(2010)^{31}\end{array}$ & 54 & Yes & Single & $\begin{array}{l}\text { Favourite music and } \\
\text { audiobook listening vs. } \\
\text { standard care / Auditory } \\
\text { sensory memory. }\end{array}$ & $\begin{array}{l}60 \text { hours in } \\
8 \text { weeks }\end{array}$ & $\begin{array}{l}\text { Listening to music and speech after neural damage can induce long-term plastic } \\
\text { changes in early sensory processing. }\end{array}$ \\
\hline $\begin{array}{l}\text { Altenmüller et al. } \\
\qquad(2009)^{33}\end{array}$ & 62 & No & No & $\begin{array}{l}\text { Children's music, folk songs, } \\
\text { and tunes vs. conventional } \\
\text { therapy / Neuroplasticity and } \\
\text { motor recovery. }\end{array}$ & $\begin{array}{l}7.5 \text { hours in } \\
3 \text { weeks }\end{array}$ & $\begin{array}{l}\text { MST improved motor skills showed by ARAT score }(p<0.001, d=0.32) \text {, Arm } \\
\text { paresis score }(p<0.05, d=0.46) \text {, Box and Block Test }(p<0.001, d=0.43) \text {, } \\
\text { and Nine Hole Pegboard Test }(p<0.05, d=0.32) .\end{array}$ \\
\hline $\begin{array}{l}\text { Särkämö et al. } \\
\quad(2008)^{32}\end{array}$ & 54 & Yes & Single & $\begin{array}{l}\text { Favourite music and } \\
\text { audiobook listening vs. } \\
\text { standard care / Cognitive } \\
\text { functions and mood. }\end{array}$ & $\begin{array}{l}60 \text { hours in } \\
8 \text { weeks }\end{array}$ & $\begin{array}{l}\text { Music listening improved verbal memory }(p=0.002, d=0.88) \text { and focused } \\
\text { attention }(p=0.012, d=0.92) \text { compared to the audiobook and control groups. } \\
\text { Music group also experienced less depression }(p=0.031, d=0.77) \text { and } \\
\text { confusion }(p=0.045, d=0.72) \text { than the control group. }\end{array}$ \\
\hline $\begin{array}{l}\text { Jeong et al. } \\
(2007)^{34}\end{array}$ & 33 & No & No & $\begin{array}{l}\text { RAS vs. referral information } \\
\text { / Upper and lower limb } \\
\text { mobility, mood, interpersonal } \\
\text { relationships, and quality of } \\
\text { life. }\end{array}$ & $\begin{array}{l}16 \text { hours in } \\
8 \text { weeks }\end{array}$ & $\begin{array}{l}\text { RAS improved range of ankle extension }(p=0.018, d=0.61) \text { and arm flexibility } \\
\text { up }(p=0.001, d=0.99) \text { and down }(p=0.008, d=0.62), \text { mood }(p=0.017, d= \\
0.03) \text {, and increased frequency and quality of interpersonal relationships }(p= \\
0.003, d=0.96) .\end{array}$ \\
\hline $\begin{array}{l}\text { Schneider et al. } \\
\qquad(2007)^{35}\end{array}$ & 40 & No & No & $\begin{array}{l}\text { Children's music, folk songs, } \\
\text { and tunes vs. conventional } \\
\text { therapy / Motor recovery. }\end{array}$ & $\begin{array}{l}7.5 \text { hours in } \\
3 \text { weeks }\end{array}$ & $\begin{array}{l}\text { Music group improved in speed, precision and smoothness of movements as } \\
\text { well as motor control in everyday activities evaluated by ARAT }(p<0.001, d= \\
\begin{array}{c}0.36) \text {, Arm paresis score }(p<0.05, d=0.42) \text {, Box and Block Test }(p<0.001, d \\
=0.69) \text {, and Nine Hole Pegboard Test }(p<0.05, d=0.24)\end{array}\end{array}$ \\
\hline $\begin{array}{l}\text { Thaut et al. } \\
(2007)^{36}\end{array}$ & 78 & No & Single & $\begin{array}{l}\text { RAS vs. } \\
\text { Neurodevelopmental } \\
\text { therapy / Gait parameters. }\end{array}$ & $\begin{array}{l}7 \cdot 5 \text { hours in } \\
3 \text { weeks }\end{array}$ & $\begin{array}{c}\text { RAS improved velocity }(p=0.006, d=2.13) \text {, stride length }(p<0.001, d=1.50) \text {, } \\
\text { cadence }(p<0.001, d=1.82) \text {, and symmetry }(p=0.049, d=0.83)\end{array}$ \\
\hline $\begin{array}{l}\text { Schauer et al. } \\
(2003)^{37}\end{array}$ & 23 & No & No & $\begin{array}{l}\text { RAS vs. gait training without } \\
\text { musical feedback / Gait } \\
\text { parameters. }\end{array}$ & $\begin{array}{l}5 \text { hours in } \\
3 \text { weeks }\end{array}$ & $\begin{array}{c}\text { RAS improved gait velocity }(p=0.008, d=0.46) \text {, stride length }(p=0.009, d= \\
0.49) \text {, cadence }(p=0.045, d=0.02), \text { symmetry }(p=0.008, d=0.55) \text {, heel-toe } \\
\text { distance }(p=0.006, d=0.40) .\end{array}$ \\
\hline $\begin{array}{l}\text { Thaut et al. } \\
(1997)^{38}\end{array}$ & 20 & No & Single & $\begin{array}{l}\text { RAS vs. physical therapy / } \\
\text { Gait parameters. }\end{array}$ & $\begin{array}{l}30 \text { hours in } \\
6 \text { weeks }\end{array}$ & $\begin{array}{c}\text { RAS improved gait velocity }(d=1.45) \text {, stride length }(d=0.93) \text {, symmetry }(d= \\
0.52) \text {, and cadence }(d=0.44) .\end{array}$ \\
\hline
\end{tabular}

16 studies

664 


\section{DEMENTIA}

\section{Sánchez et al. \\ $(2016)^{5}$ \\ $(2016)^{44}$ \\ Raglio et al \\ $(2015)^{45}$ \\ Hsu et al.}

18

Särkämö et al.

$(2015)^{46}$

Särkämö et al.

$(2015)^{47}$

Chu et al.
$(2014)^{58}$

Vink et al.

$(2014)^{48}$

Narme et al

$(2014)^{49}$
No

No

Multisensory stimulation vs.

$$
\text { music listening / }
$$

Neuropsychiatric symptoms and cognition.

Music listening and/or

singing vs. standard care /

Emotional parameters.

Music therapy and music listening vs. standard care / Behavioral and psychological symptoms of dementia.

Music listening, singing, improvising and talking vs. standard care /

Neuropsychiatric symptoms, well-being, and carerresident interaction.

Singing or music listening vs. standard care / Clinical, demographic, and musical background factors

influencing the cognitive and emotional efficacy of caregiver-implemented musical activities.

Group music therapy vs. standard care / Mood and cognition.

Music therapy (listening and singing) vs. other activities / Neuropsychiatric symptoms.

Music therapy (listening, playing and singing) vs. cooking / Patients' mood

cognition, behavioral disturbances, and on the and stress experienced by nurses.

Särkämö et al

83

Yes

Single

Singing or music listening vs. standard care / Quality of life, mood and cognition.

Vink et al.

$(2013)^{50}$

Ceccato et al.

$(2012)^{51}$

Sung et al.

$(2012)^{52}$

Lin et al.

$(2011)^{53}$

Cooke et al.

$(2010)^{60}$

Raglio et al.

$(2010)^{54}$

Guétin et al.

$(2009)^{55}$
77

50

52

100

47

60

30

50

Music listening and singing vs. other activites / Agitation.

Music therapy vs. standard care / Cognition and anxiety.

Favourite music vs. standard care / Anxiety.

Music therapy (playing and listening) vs. standard care / Agitation.

Music therapy (listening and playing) vs. reading / Mood and quality of life.

Music therapy vs. standard

$$
\begin{aligned}
& \text { care / Behavioral } \\
& \text { disturbances. }
\end{aligned}
$$

Music therapy vs. resting and reading / Anxiety and mood.
16 hours in

16 weeks

15 hours in

10 weeks

10 hours in

10 weeks

11 hours in

22 weeks

15 hours in

10 weeks

6 hours in

6 weeks

21 hours in

16 weeks

8 hours in

4 weeks

15 hours in

10 weeks

21 hours in

16 weeks

18 hours in

12 weeks

6 hours in

6 weeks

6 hours in

6 weeks

32 hours in

16 weeks

6 hours in

4 weeks

5 hours in

16 weeks
Multisensory stimulation showed positive effects on anxiety symptoms and dementia severity that were not observed in the music group.

Both music listening and singing groups improved in behavioral disturbances ( $p$ $=0.04, d=0.42)$ and physical signs $(p=0.008, d=0.52)$ more than the contro group 6 months after the intervention, found effects were not present anymore.

There were no significant differences between the groups.

Music group showed improvement in symptoms $(p=0.002, d=2.32)$ and in levels of wellbeing $(p<0.001, d=3.85)$. Staff in the intervention group reported enhanced caregiving techniques as a result of the programme.

Singing was beneficial especially in improving working memory in mild dementia and in maintaining executive function and orientation in younger PWDs. Music listening was beneficial in supporting general cognition, working memory, and quality of life especially in moderate dementia not caused by Alzheimer's disease $(A D)$ who were in institutional care. Both music interventions alleviated depression especially in mild dementia and AD. The musical background of the PWD did not influence the efficacy of the music interventions.

Group music therapy decreased depression $(p=0.001, d=0.21)$ and delayed the deterioration of cognitive functions, especially recall $(p=0.004, d=0.72)$. The effects were present 1 month after cessation of the intervention.

Neuropsychiatric symptoms decreased significantly in the music therapy group $(p=0.01)$.

There were no significant differences between the groups.

Music listening improved the patients' $\operatorname{mood}(p=0.001, d=0.80)$, orientation ( $=0.005, d=0.71)$, episodic memory $(p=0.036, d=0.54)$ attention and executive functions $(p=0.039, d=0.48)$, overall cognitive performance $(p=$ $0.041, d=0.47)$, and the quality of life $(p<0.001, d=0.99)$. Singing resulted in additional improvement in short-term memory and working memory $(p=0.006$, $d=0 \cdot 75)$, and improved the caregiver wellbeing $(p=0.026, d=0.85)$.

There were no significant differences between the groups.

The music group improved performance in attention $(p=0.001, d=0.76)$ and verbal episodic memory tasks (immediate $p=0.001 d=0.76$, delayed $p=0.001$ $d=0.73)$, but not in anxiety.

Anxiety decreased in the music group ( $p=0.004, d=0.06$ ).

There were no significant differences between the groups.

There were no significant differences between the groups.

Music reduced the behavioral disturbances showed by significant group difference $(p<0.05, d=0.63)$.

Music therapy decreased anxiety $(p<0.001, d=2.42)$ and depression $(p=$ $0 \cdot 002, d=1 \cdot 05$ ). These effects persisted up to 2 months after stopping the intervention. 


\begin{tabular}{|c|c|c|c|c|c|c|}
\hline $\begin{array}{c}\text { Raglio et al. } \\
(2008)^{56}\end{array}$ & 59 & Yes & Single & $\begin{array}{l}\text { Music therapy vs. other } \\
\text { activites / Behavioral and } \\
\text { psychologic symptoms. }\end{array}$ & $\begin{array}{c}15 \text { hours in } \\
16 \text { weeks }\end{array}$ & $\begin{array}{c}\text { Music therapy improved behavioral symptoms }(p<0.0001, d=1.04) \text {, functional } \\
\text { ability }(p<0.0001, d=0.79) \text {, and empathetic behavior }(p<0.0001, d=0.61) \\
\text { compared to the control treatment. }\end{array}$ \\
\hline
\end{tabular}

Number of participants MT involved Blinding $\begin{gathered}\text { Study design / Primary } \\ \text { outcome }\end{gathered}$ Overall intervention time

\begin{tabular}{|c|c|c|c|c|c|c|}
\hline \multicolumn{7}{|c|}{ PARKINSON'S DISEASE (PD) } \\
\hline $\begin{array}{l}\text { Pohl et al. } \\
(2013)^{63}\end{array}$ & 18 & Yes & Single & $\begin{array}{l}\text { Music listening, rhythmic } \\
\text { clapping or stomping vs. } \\
\text { standard care / Motor } \\
\text { performance, cognition, } \\
\text { quality of life }\end{array}$ & $\begin{array}{l}12 \text { hours in } \\
6 \text { weeks }\end{array}$ & $\begin{array}{l}\text { Music therapy improved mobility }(p=0.006) \text {, UPDRS }(p=0.003) \text {, Text re-call ( } p \\
=0.036) \text {, Item naming }(p=0.033) \text {, performance in Stroop test }(p=0.007) \text {, and } \\
\text { Quality of life }(p=0.031) .\end{array}$ \\
\hline $\begin{array}{l}\text { de Bruin et al. } \\
(2010)^{64}\end{array}$ & 22 & No & Single & $\begin{array}{l}\text { Favorite music synchronized } \\
\text { to gait vs. regular activities / } \\
\text { Walking parameters }\end{array}$ & $\begin{array}{l}19 \cdot 5 \text { hours in } \\
13 \text { weeks }\end{array}$ & $\begin{array}{l}\text { Walking to music improved velocity }(p=0.002, d=2 \cdot 64) \text {, stride time }(p=0.019 \text {, } \\
\qquad d=1 \cdot 76) \text {, cadence }(p=0.007, d=2 \cdot 16), \text { UPDRS }(p=0.002, d=0.50) \text {. }\end{array}$ \\
\hline $\begin{array}{l}\text { Hackney et al. } \\
\qquad(2009)^{65}\end{array}$ & 48 & No & Single & $\begin{array}{l}\text { Tango or waltz/foxtrot vs. } \\
\text { standard care / Functional } \\
\text { motor control. }\end{array}$ & $\begin{array}{l}20 \text { hours in } \\
13 \text { weeks }\end{array}$ & $\begin{array}{l}\text { Tango group improved in balance }(p=0.001, d=2.98), 6 \text {-minute walking }(p= \\
0.001, d=2.50) \text { and backward stride length }(p=0.001, d=2 \cdot 19) \text { and } \\
\text { Waltz/Foxtrot group in balance }(p=0.001, d=3 \cdot 17), 6 \text {-minute walking }(p= \\
0.001, d=2 \cdot 24) \text { and backward stride length }(p=0.018, d=1.96) \text {. }\end{array}$ \\
\hline $\begin{array}{l}\text { Hackney et al. } \\
\qquad(2009)^{67}\end{array}$ & 61 & No & No & $\begin{array}{l}\text { Tango and waltz/foxtrot vs. } \\
\text { Tai Chi or standard care / } \\
\text { Health-related quality of life. }\end{array}$ & $\begin{array}{l}20 \text { hours in } \\
13 \text { weeks }\end{array}$ & $\begin{array}{c}\text { Tango improved mobility }(p=0.03, d=2.50) \text {, social support }(p=0.05, d= \\
2.97) \text {, and Quality of life }(p<0.01, d=2.09)\end{array}$ \\
\hline $\begin{array}{l}\text { Hackney et al. } \\
(2007)^{66}\end{array}$ & 19 & No & Single & $\begin{array}{l}\text { Tango vs. physical exercise } \\
\text { / Functional mobility. }\end{array}$ & $\begin{array}{l}20 \text { hours in } \\
13 \text { weeks }\end{array}$ & Tango group improved in balance $(p=0.01, d=2 \cdot 18)$ \\
\hline
\end{tabular}

5 studies 168

Number of participants MT involved Blinding $\begin{gathered}\text { Study design / Primary } \\ \text { outcome }\end{gathered} \quad$ Overall intervention time

\begin{tabular}{|c|c|c|c|c|c|c|}
\hline \multicolumn{7}{|c|}{ MULTIPLE SCLEROSIS (MS) } \\
\hline $\begin{array}{l}\text { Gatti et al. } \\
(2015)^{69}\end{array}$ & 19 & No & No & $\begin{array}{l}\text { Keyboard playing vs. mute } \\
\text { keyboard playing / Hand } \\
\text { function. }\end{array}$ & $\begin{array}{l}7.5 \text { hours } \\
\text { in } 2 \text { weeks }\end{array}$ & $\begin{array}{l}\text { The music group improved in the functional use of the hand significantly more } \\
\text { showed by Time } \times \text { Group interaction }(p=0 \cdot 003, d=0 \cdot 60) \text {. }\end{array}$ \\
\hline $\begin{array}{l}\text { Conklyn et al. } \\
\qquad(2010)^{70}\end{array}$ & 10 & Yes & No & $\begin{array}{l}\text { RAS vs. standard care / Gait } \\
\text { parameters. }\end{array}$ & 2 weeks & $\begin{array}{l}\text { RAS significantly decreased double-support time (left: } p=0.018, d=1.61 \text {; right: } \\
\qquad p=0.025, d=1.46)\end{array}$ \\
\hline
\end{tabular}

2 studies

29

Number of participants MT involved Blinding $\begin{gathered}\text { Study design / Primary } \\ \text { outcome }\end{gathered} \quad$ Overall intervention time

EPILEPSY

\begin{tabular}{|c|c|c|c|c|c|c|}
\hline $\begin{array}{l}\text { Bodner et al. } \\
\quad(2012)^{73}\end{array}$ & 73 & No & Single & $\begin{array}{l}\text { Jightly exposure of Mozart } \\
\text { Sonata K. } 448 \text { vs. no } \\
\text { intervention / Seizure } \\
\text { occurrence. }\end{array}$ & $\begin{array}{l}\text { Every night } \\
\text { for } 1 \text { year }\end{array}$ & $\begin{array}{l}\text { Music group significantly decreased in seizure frequency during the treatment } \\
\text { phase }(17 \%, p=0.014) \text { and even one year post-treatment }(16 \%, p=0.027) \text {. }\end{array}$ \\
\hline
\end{tabular}

1 study

73

Effect size $=$ mean pre-post change in the treatment group minus the mean pre-post change in the control group, divided by the pooled pre-test standard deviation ${ }^{96}$

Effect size was defined small when $d=0.2$, medium when $d=0.5$ and large when $d=0.8$.

$d=$ Effect size, BATRAC = Bilateral arm training with rhythmic auditory cueing, MIT = Melodic Intonation Therapy, MST $=$ Music-supported Therapy, MT = Music therapist, RAS = Rhythmic auditory stimulation. 


\section{References}

482 1. World Health Organization (WHO). World report on ageing and health. 2015.

483 Available at:

484 http://apps.who.int/iris/bitstream/10665/186463/1/9789240694811_eng.pdf?ua=1.

485 Accessed 04/11, 2017.

486 2. PricewaterhouseCoopers Health Industries. The annual cost of brain disease 2012.

487 2012. Available at: http://pwchealth.com/cgi-local/hregister.cgi/reg/annual-cost-of-brain488 disease-2012.pdf. Accessed 04/11, 2017.

489 3. Olesen J, Gustavsson A, Svensson M, Wittchen H-, Jönsson B. The economic cost of 490 brain disorders in Europe. European Journal of Neurology 2012; 19(1): 155-62.

491 4. Nudo RJ. Recovery after brain injury: mechanisms and principles. Frontiers in Human 492 Neuroscience 2013; 7.

493 5. Tomassini V, Matthews PM, Thompson AJ, et al. Neuroplasticity and functional 494 recovery in multiple sclerosis. Nat Rev Neurol 2012; 8(11): 635-46.

495 6. Hill NL, Kolanowski AM, Gill DJ. Plasticity in early Alzheimer's disease: an opportunity 496 for intervention. Top Geriatr Rehabil 2011; 27(4): 257-67.

497 7. Reetz K, Tadic V, Kasten M, et al. Structural imaging in the presymptomatic stage of 498 genetically determined parkinsonism. Neurobiol Dis 2010; 39(3): 402-8.

499 8. Cramer SC, Sur M, Dobkin BH, et al. Harnessing neuroplasticity for clinical 500 applications. Brain 2011; 134(Pt 6): 1591-609. 
501 9. Zeiler SR, Krakauer JW. The interaction between training and plasticity in the 502 poststroke brain. Curr Opin Neurol 2013; 26(6): 609-16.

503 10. Herholz SC, Herholz RS, Herholz K. Non-pharmacological interventions and 504 neuroplasticity in early stage Alzheimer's disease. Expert Rev Neurother 2013; 13(11): $505 \quad 1235-45$.

506 11. Agosta F, Gatti R, Sarasso E, et al. Brain plasticity in Parkinson's disease with 507 freezing of gait induced by action observation training. J Neurol 2017; 264(1): 88-101.

508 12. Enzinger C, Pinter D, Rocca MA, De Luca J, Sastre-Garriga J, Audoin B, Filippi M. 509 Longitudinal fMRI studies: Exploring brain plasticity and repair in MS. Mult Scler 2016; 510 22(3): 269-78.

511 13. Baroncelli L, Braschi C, Spolidoro M, Begenisic T, Sale A, Maffei L. Nurturing brain 512 plasticity: impact of environmental enrichment. Cell Death Differ 2010; 17(7): 1092-103.

513 14. Zatorre RJ, Chen JL, Penhune VB. When the brain plays music: auditory-motor 514 interactions in music perception and production. Nature Reviews Neuroscience 2007; 8(7): $515 \quad 547-58$.

516 15. Koelsch S. Brain correlates of music-evoked emotions. Nat Rev Neurosci 2014; 517 15(3): 170-80.

518 16. Särkämö T, Tervaniemi M, Huotilainen M. Music perception and cognition: 519 development, neural basis, and rehabilitative use of music. Wiley Interdisciplinary 520 Reviews: Cognitive Science 2013; 4(4): 441-51. 
521 17. Alluri V, Toiviainen P, Jääskeläinen IP, Glerean E, Sams M, Brattico E. Large-scale 522 brain networks emerge from dynamic processing of musical timbre, key and rhythm.

523 Neuroimage 2012; 59(4): 3677-89.

524 18. Wan CY, Schlaug G. Music making as a tool for promoting brain plasticity across the 525 life span. Neuroscientist 2010; 16(5): 566-77.

526 19. Schlaug G. Musicians and music making as a model for the study of brain plasticity. 527 Prog Brain Res 2015; 217: 37-55.

528 20. Vaquero L, Hartmann K, Ripollés P, et al. Structural neuroplasticity in expert pianists 529 depends on the age of musical training onset. Neuroimage 2016; 1(126): 106-19.

530 21. Hole J, Hirsch M, Ball E, Meads C. Music as an aid for postoperative recovery in 531 adults: a systematic review and meta-analysis. Lancet 2015; 386(10004): 1659-71.

532 22. Magee WL, Clark I, Tamplin J, Bradt J. Music interventions for acquired brain injury. 533 Cochrane Database Syst Rev 2017; 1: CD006787.

534 23. Benjamin EJ, Blaha MJ, Chiuve SE, et al. Heart Disease and Stroke Statistics-2017 535 Update: A Report From the American Heart Association. Circulation 2017; 135(10): e146536603.

537 24. van Vugt FT, Kafczyk T, Kuhn W, Rollnik JD, Tillmann B, Altenmüller E. The role of 538 auditory feedback in music-supported stroke rehabilitation: A single-blinded randomised 539 controlled intervention. Restor Neurol Neurosci 2016; 34(2): 297-311. 
540 25. Scholz DS, Rohde S, Nikmaram N, Bruckner HP, Grossbach M, Rollnik JD,

541 Altenmüller EO. Sonification of Arm Movements in Stroke Rehabilitation - A Novel

542 Approach in Neurologic Music Therapy. Front Neurol 2016; 7: 106.

543 26. Raglio A, Oasi O, Gianotti M, Rossi A, Goulene K, Stramba-Badiale M. Improvement

544 of spontaneous language in stroke patients with chronic aphasia treated with music

545 therapy: a randomized controlled trial. Int J Neurosci 2016; 126(3): 235-42.

546 27. Tong Y, Forreider B, Sun X, et al. Music-supported therapy (MST) in improving post547 stroke patients' upper-limb motor function: a randomised controlled pilot study. Neurol Res $548 \quad 2015 ; 37(5): 434-40$.

549 28. Särkämö T, Ripollés $\mathrm{P}$, Vepsäläinen $\mathrm{H}$, et al. Structural changes induced by daily 550 music listening in the recovering brain after middle cerebral artery stroke: a voxel-based 551 morphometry study. Front Hum Neurosci 2014; 8: 245.

552 29. van der Meulen I, van de Sandt-Koenderman WM, Heijenbrok-Kal MH, Visch-Brink 553 EG, Ribbers GM. The Efficacy and Timing of Melodic Intonation Therapy in Subacute 554 Aphasia. Neurorehabil Neural Repair 2014; 28(6): 536-44.

555 30. Cha Y, Kim Y, Hwang S, Chung Y. Intensive gait training with rhythmic auditory 556 stimulation in individuals with chronic hemiparetic stroke: a pilot randomized controlled 557 study. NeuroRehabilitation 2014; 35(4): 681-8.

558 31. Särkämö T, Pihko E, Laitinen S, et al. Music and Speech Listening Enhance the 559 Recovery of Early Sensory Processing after Stroke. J Cogn Neurosci 2010; 22(12): 271656027. 
561 32. Särkämö T, Tervaniemi M, Laitinen S, et al. Music listening enhances cognitive 562 recovery and mood after middle cerebral artery stroke. Brain 2008; 131: 866-76.

563 33. Altenmüller E, Marco-Pallares J, Münte TF, Schneider S. Neural reorganization 564 underlies improvement in stroke-induced motor dysfunction by music-supported therapy. 565 Ann N Y Acad Sci 2009; 1169: 395-405.

566 34. Jeong S, Kim MT. Effects of a theory-driven music and movement program for 567 stroke survivors in a community setting. Appl Nurs Res 2007; 20(3): 125-31.

568 35. Schneider S, Schoenle PW, Altenmüller E, Muente TF. Using musical instruments to 569 improve motor skill recovery following a stroke. J Neurol 2007; 254(10): 1339-46.

570 36. Thaut MH, Leins AK, Rice RR, et al. Rhythmic auditory stimulation improves gait 571 more than NDT/Bobath training in near-ambulatory patients early poststroke: a single572 blind, randomized trial. Neurorehabil Neural Repair 2007; 21(5): 455-9.

573 37. Schauer M, Mauritz K. Musical motor feedback (MMF) in walking hemiparetic stroke 574 patients: randomized trials of gait improvement. Clin Rehabil 2003; 17(7): 713-22.

575 38. Thaut MH, Mclntosh GC, Rice RR. Rhythmic facilitation of gait training in 576 hemiparetic stroke rehabilitation. J Neurol Sci 1997; 151(2): 207-12.

577 39. Whitall J, Waller SM, Sorkin JD, et al. Bilateral and unilateral arm training improve 578 motor function through differing neuroplastic mechanisms: a single-blinded randomized 579 controlled trial. Neurorehabil Neural Repair 2011; 25(2): 118-29. 
580 40. Stroke Association. State of the Nation. Stroke statistics - January 2016. 2015.

581 Available at: https://www.stroke.org.uk/sites/default/files/stroke_statistics_2015.pdf.

582 Accessed 04/11, 2017.

583 41. Albert ML, Sparks RW, Helm NA. Melodic intonation therapy for aphasia. Arch 584 Neurol 1973; 29(2): 130-1.

585 42. Nys GM, van Zandvoort MJ, de Kort PL, Jansen BP, de Haan EH, Kappelle LJ.

586 Cognitive disorders in acute stroke: prevalence and clinical determinants. Cerebrovasc Dis $5872007 ; 23(5-6): 408-16$.

588 43. Hackett ML, Pickles K. Part I: frequency of depression after stroke: an updated 589 systematic review and meta-analysis of observational studies. Int J Stroke 2014; 9(8): $590 \quad 1017-25$.

591 44. Särkämö T, Laitinen S, Numminen A, Kurki M, Johnson JK, Rantanen P. Pattern of 592 Emotional Benefits Induced by Regular Singing and Music Listening in Dementia. J Am 593 Geriatr Soc 2016; 64(2): 439-40.

594 45. Raglio A, Bellandi D, Baiardi P, et al. Effect of Active Music Therapy and 595 Individualized Listening to Music on Dementia: A Multicenter Randomized Controlled Trial. $596 J$ J Am Geriatr Soc 2015; 63(8): 1534-9.

597 46. Hsu MH, Flowerdew R, Parker M, Fachner J, Odell-Miller H. Individual music 598 therapy for managing neuropsychiatric symptoms for people with dementia and their 599 carers: a cluster randomised controlled feasibility study. BMC Geriatr 2015; 15: 84,015$600 \quad 0082-4$. 
601 47. Särkämö T, Laitinen S, Numminen A, Kurki M, Johnson JK, Rantanen P. Clinical 602 and Demographic Factors Associated with the Cognitive and Emotional Efficacy of 603 Regular Musical Activities in Dementia. J Alzheimers Dis 2015; 49(3): 767-81.

604 48. Vink AC, Zuidersma M, Boersma F, de Jonge P, Zuidema SU, Slaets JP. Effect of 605 music therapy versus recreational activities on neuropsychiatric symptoms in elderly adults 606 with dementia: an exploratory randomized controlled trial. J Am Geriatr Soc 2014; 62(2): $607 \quad 392-3$.

608 49. Narme P, Clement S, Ehrle N, et al. Efficacy of musical interventions in dementia: 609 evidence from a randomized controlled trial. J Alzheimers Dis 2014; 38(2): 359-69.

610 50. Vink AC, Zuidersma M, Boersma F, de Jonge P, Zuidema SU, Slaets JP. The effect 611 of music therapy compared with general recreational activities in reducing agitation in 612 people with dementia: a randomised controlled trial. Int J Geriatr Psychiatry 2013; 28(10): $613 \quad 1031-8$.

614 51. Ceccato E, Vigato G, Bonetto C, et al. STAM protocol in dementia: a multicenter, 615 single-blind, randomized, and controlled trial. Am J Alzheimers Dis Other Demen 2012; 616 27(5): 301-10.

617 52. Sung HC, Lee WL, Li TL, Watson R. A group music intervention using percussion 618 instruments with familiar music to reduce anxiety and agitation of institutionalized older 619 adults with dementia. Int J Geriatr Psychiatry 2012; 27(6): 621-7.

620 53. Lin $\mathrm{Y}, \mathrm{Chu} \mathrm{H}$, Yang $\mathrm{CY}$, et al. Effectiveness of group music intervention against 621 agitated behavior in elderly persons with dementia. Int J Geriatr Psychiatry 2011; 26(7): $622670-8$. 
623 54. Raglio A, Bellelli G, Traficante D, et al. Efficacy of music therapy treatment based on 624 cycles of sessions: a randomised controlled trial. Aging Ment Health 2010; 14(8): 900-4.

625 55. Guetin S, Portet F, Picot MC, et al. Effect of music therapy on anxiety and 626 depression in patients with Alzheimer's type dementia: randomised, controlled study. 627 Dement Geriatr Cogn Disord 2009; 28(1): 36-46.

628 56. Raglio A, Bellelli G, Traficante D, Gianotti M, Ubezio MC, Villani D, Trabucchi M. 629 Efficacy of music therapy in the treatment of behavioral and psychiatric symptoms of 630 dementia. Alzheimer Dis Assoc Disord 2008; 22(2): 158-62.

631 57. Sánchez A, Maseda A, Marante-Moar MP, de Labra C, Lorenzo-López L, Millán632 Calenti JC. Comparing the Effects of Multisensory Stimulation and Individualized Music 633 Sessions on Elderly People with Severe Dementia: A Randomized Controlled Trial. J 634 Alzheimers Dis 2016; 52(1): 303-15.

635 58. Chu H, Yang CY, Lin Y, Ou KL, Lee TY, O'Brien AP, Chou KR. The impact of group 636 music therapy on depression and cognition in elderly persons with dementia: a 637 randomized controlled study. Biol Res Nurs 2014; 16(2): 209-17.

638 59. Särkämö T, Tervaniemi M, Laitinen S, Numminen A, Kurki M, Johnson JK, 639 Rantanen P. Cognitive, emotional, and social benefits of regular musical activities in early 640 dementia: randomized controlled study. Gerontologist 2014; 54(4): 634-50.

641 60. Cooke ML, Moyle W, Shum DH, Harrison SD, Murfield JE. A randomized controlled 642 trial exploring the effect of music on agitated behaviours and anxiety in older people with 643 dementia. Aging Ment Health 2010; 14(8): 905-16. 
644 61. Hall CB, Lipton RB, Sliwinski M, Katz MJ, Derby CA, Verghese J. Cognitive activities 645 delay onset of memory decline in persons who develop dementia. Neurology 2009; 73(5): $646 \quad 356-61$.

647 62. Hanagasi HA, Tufekcioglu Z, Emre M. Dementia in Parkinson's disease. J Neurol 648 Sci 2017; 374: 26-31.

649 63. Pohl P, Dizdar N, Hallert E. The Ronnie Gardiner Rhythm and Music Method - a 650 feasibility study in Parkinson's disease. Disabil Rehabil 2013; 35(26): 2197-204.

651 64. de Bruin N, Doan JB, Turnbull G, Suchowersky O, Bonfield S, Hu B, Brown LA. 652 Walking with music is a safe and viable tool for gait training in Parkinson's disease: the 653 effect of a 13-week feasibility study on single and dual task walking. Parkinsons Dis 2010; 654 2010: 483530.

655 65. Hackney ME, Earhart GM. Effects of dance on movement control in Parkinson's 656 disease: a comparison of Argentine tango and American ballroom. J Rehabil Med 2009; 657 41(6): 475-81.

658 66. Hackney ME, Kantorovich S, Levin R, Earhart GM. Effects of tango on functional 659 mobility in Parkinson's disease: a preliminary study. J Neurol Phys Ther 2007; 31(4): 1736609.

661 67. Hackney ME, Earhart GM. Health-related quality of life and alternative forms of 662 exercise in Parkinson disease. Parkinsonism Relat Disord 2009; 15(9): 644-8.

663 68. Nombela C, Hughes LE, Owen AM, Grahn JA. Into the groove: can rhythm influence 664 Parkinson's disease? Neurosci Biobehav Rev 2013; 37(10 Pt 2): 2564-70. 
665 69. Gatti R, Tettamanti A, Lambiase S, Rossi P, Comola M. Improving hand functional 666 use in subjects with multiple sclerosis using a musical keyboard: a randomized controlled 667 trial. Physiother Res Int 2015; 20(2): 100-7.

668 70. Conklyn D, Stough D, Novak E, Paczak S, Chemali K, Bethoux F. A home-based 669 walking program using rhythmic auditory stimulation improves gait performance in patients 670 with multiple sclerosis: a pilot study. Neurorehabil Neural Repair 2010; 24(9): 835-42.

671 71. Rao AK, Mazzoni P, Wasserman P, Marder K. Longitudinal Change in Gait and 672 Motor Function in Pre-manifest Huntington's Disease. PLoS Curr 2011; 3: RRN1268.

673 72. Fisher RS. Therapeutic devices for epilepsy. Ann Neurol 2012; 71(2): 157-68.

674 73. Bodner M, Turner RP, Schwacke J, Bowers C, Norment C. Reduction of seizure 675 occurrence from exposure to auditory stimulation in individuals with neurological 676 handicaps: a randomized controlled trial. PLoS One 2012; 7(10): e45303.

677 74. Dastgheib SS, Layegh P, Sadeghi R, Foroughipur M, Shoeibi A, Gorji A. The effects 678 of Mozart's music on interictal activity in epileptic patients: systematic review and meta679 analysis of the literature. Curr Neurol Neurosci Rep 2014; 14(1): 420,013-0420-x.

680 75. Meyer GF, Spray A, Fairlie JE, Uomini NT. Inferring common cognitive mechanisms 681 from brain blood-flow lateralization data: a new methodology for fTCD analysis. Front 682 Psychol 2014; 5: 552.

683 76. Murphy TH, Corbett D. Plasticity during stroke recovery: from synapse to behaviour. 684 Nat Rev Neurosci 2009; 10(12): 861-72. 
685 77. Grau-Sanchez J, Amengual JL, Rojo N, et al. Plasticity in the sensorimotor cortex 686 induced by Music-supported therapy in stroke patients: a TMS study. Front Hum Neurosci $687 \quad 2013 ; 7: 494$.

688 78. Ripollés P, Rojo N, Grau-Sanchez J, et al. Music supported therapy promotes motor 689 plasticity in individuals with chronic stroke. Brain Imaging Behav 2015.

690 79. Amengual JL, Rojo N, Veciana de Las Heras M, et al. Sensorimotor plasticity after 691 music-supported therapy in chronic stroke patients revealed by transcranial magnetic 692 stimulation. PLoS One 2013; 8(4): e61883.

693 80. Schlaug G, Marchina S, Norton A. Evidence for Plasticity in White-Matter Tracts of 694 Patients with Chronic Broca's Aphasia Undergoing Intense Intonation-based Speech 695 Therapy. Neurosciences and Music lii: Disorders and Plasticity 2009; 1169: 385-94.

696 81. Salimpoor VN, Benovoy M, Larcher K, Dagher A, Zatorre RJ. Anatomically distinct 697 dopamine release during anticipation and experience of peak emotion to music. Nat 698 Neurosci 2011; 14(2): 257-62.

699 82. Okada K, Kurita A, Takase B, et al. Effects of music therapy on autonomic nervous 700 system activity, incidence of heart failure events, and plasma cytokine and catecholamine 701 levels in elderly patients with cerebrovascular disease and dementia. Int Heart J 2009; $70250(1): 95-110$.

703 83. Bradt J, Dileo C, Potvin N. Music for stress and anxiety reduction in coronary heart 704 disease patients. Cochrane Database Syst Rev 2013; (12):CD006577. doi(12): 705 CD006577. 
706 84. Radley J, Morilak D, Viau V, Campeau S. Chronic stress and brain plasticity:

707 Mechanisms underlying adaptive and maladaptive changes and implications for stress-

708 related CNS disorders. Neurosci Biobehav Rev 2015; 58: 79-91.

709 85. Barugh AJ, Gray P, Shenkin SD, Maclullich AM, Mead GE. Cortisol levels and the

710 severity and outcomes of acute stroke: a systematic review. J Neurol 2014; 261(3): 533-

71145.

712 86. Nilsson U. Soothing music can increase oxytocin levels during bed rest after open-

713 heart surgery: a randomised control trial. J Clin Nurs 2009; 18(15): 2153-61.

714 87. Nilsson $\mathrm{U}$. The effect of music intervention in stress response to cardiac surgery in a 715 randomized clinical trial. Heart \& Lung 2009; 38(3): 201-7.

716 88. Raglio A, Attardo L, Gontero G, Rollino S, Groppo E, Granieri E. Effects of music

717 and music therapy on mood in neurological patients. World J Psychiatry 2015; 5(1): 68-78.

718 89. Pan A, Sun Q, Okereke OI, Rexrode KM, Hu FB. Depression and risk of stroke

719 morbidity and mortality: a meta-analysis and systematic review. JAMA 2011; 306(11):

$720 \quad 1241-9$

721 90. Towfighi A, Ovbiagele B, El Husseini N, et al. Poststroke Depression: A Scientific

722 Statement for Healthcare Professionals From the American Heart Association/American

723 Stroke Association. Stroke 2017; 48(2): e30-43.

724 91. Zarate JM. The neural control of singing. Front Hum Neurosci 2013; 7: 237. 
725 92. Wan CY, Zheng X, Marchina S, Norton A, Schlaug G. Intensive therapy induces 726 contralateral white matter changes in chronic stroke patients with Broca's aphasia. Brain 727 Lang 2014; 136: 1-7.

728 93. Dahlin O. Beräattelse om en dumbe, som kan siumga (On a Mute Who Can Sing). 729 Kungl. Svenska Vetensk. Acad. Handl. 1745; 6: 114-5.

730 94. Jacobsen JH, Stelzer J, Fritz TH, Chetelat G, La Joie R, Turner R. Why musical 731 memory can be preserved in advanced Alzheimer's disease. Brain 2015; 138(Pt 8): 243873250.

733 95. Foley N, McClure JA, Meyer M, Salter K, Bureau Y, Teasell R. Inpatient 734 rehabilitation following stroke: amount of therapy received and associations with functional 735 recovery. Disabil Rehabil 2012; 34(25): 2132-8.

736 96. Morris SB. Estimating Effect Sizes From Pretest-Posttest-Control Group Designs. 737 Organ Res Methods 2008; 11(2): 364-86. 